\title{
Markov Set-Chains as abstractions of Stochastic Hybrid Systems *
}

\author{
Alessandro Abate ${ }^{1}$, Alessandro D'Innocenzo ${ }^{2}$, \\ Maria D. Di Benedetto ${ }^{2}$, and Shankar S. Sastry ${ }^{3}$ \\ 1 Department of Aeronautics and Astronautics, Stanford University - USA \\ aabate@stanford.edu \\ 2 Department of Electrical Engineering and Computer Science, \\ Center of Excellence DEWS, University of L'Aquila - Italy \\ \{adinnoce, dibenede\}@ing. univaq.it \\ 3 Department of Electrical Engineering and Computer Sciences, \\ University of California, Berkeley - USA \\ sastry@eecs.berkeley.edu
}

\begin{abstract}
The objective of this study is to introduce an abstraction procedure that applies to a general class of dynamical systems, that is to discrete-time stochastic hybrid systems (dt-SHS). The procedure abstracts the original dt-SHS into a Markov set-chain (MSC) in two steps. First, a Markov chain (MC) is obtained by partitioning the hybrid state space, according to a controllable parameter, into non-overlapping domains and computing transition probabilities for these domains according to the dynamics of the dt-SHS. Second, explicit error bounds for the abstraction that depend on the above parameter are derived, and are associated to the computed transition probabilities of the MC, thus obtaining a MSC. We show that one can arbitrarily increase the accuracy of the abstraction by tuning the controllable parameter, albeit at an increase of the cardinality of the MSC. Resorting to a number of results from the MSC literature allows the analysis of the dynamics of the original dt-SHS. In the present work, the asymptotic behavior of the dt-SHS dynamics is assessed within the abstracted framework.
\end{abstract}

\section{Introduction and Objectives}

Hybrid Systems (HS) are dynamical systems with interleaved continuous and discrete behaviors. Their great expressive power is offset by two main issues. The first is the subtlety of their theoretical investigation: much research has been directed to further the understanding of their system-theoretical properties. The second is the problem of scalability, in particular with respect to computational complexity. For instance, the formal verification of properties of the system (e.g. model checking techniques [4]) is complicated by the continuity of the state-space and by the interaction between continuous and discrete dynamics.

A technique which is often employed to cope with system complexity and dimensionality is abstraction. According to this approach, a system with a smaller

\footnotetext{
* This work was partially supported by European Commission under Project IST NoE HYCON contract n. 511368, STREP project n. TREN/07/FP6AE/S07.71574/037180 IFLY, and by the NSF grant CCR-0225610.
} 
state space (possibly finite) is obtained, which is equivalent to the system under study. Systems equivalence is usually defined via the notions of language equivalence and bisimulation [2]. Recently, approximate notions of system equivalence [7] have been developed, where a metric is introduced to quantify the distance between the original system and the abstraction. The contribution in [6] proposes an algorithm to construct an approximate abstraction of a HS by means of a timed automaton. In [9] a notion of approximate bisimilarity is proposed for a class of Stochastic Hybrid Systems (SHS), that is HS which are endowed with probabilistic terms.

The present contribution introduces a formal abstraction procedure for a general class of SHS. This work refers to a discrete time framework and introduces the explicit presence of spatial guards in a class of SHS (named dt-SHS), and shows that it is possible to express the transition probability function in a compact way by employing the concept of probabilistic reachability. After introducing a partitioning procedure on the hybrid state space, the transition probabilities between these partitions are approximately computed, thus generating a Markov chain (MC). By raising some continuity assumptions on the entities that characterize the dynamics of the dt-SHS, explicit error bounds are associated to the transition probabilities. These error bounds depend on the diameter of the introduced partitions and can then be refined by this parameter. This allows to formally set up a Markov set-chain (MSC) associated to the partitioning procedure. The asymptotic behavior of the MSC is then related to that of the dt-SHS.

The present technique is analogous to the line of work presented in [10], which proposes a discretization of the continuous dynamics of a Markov process into that of a MC, defined on a grid on the state-space. The contribution shows weak convergence of the MC process to the original one, but no error bounds are explicitly derived. Both this work and [10] approximate the original process with a probabilistic discrete structure. This provides a connection to model checking of stochastic timed automata (which is a subclass of SHS), that has been investigated in [3]. A general understanding of the area of probabilistic model checking for SHS is however still far. As a first result towards this goal, we have shown the ability to construct a finite state abstraction that possibly allows us to efficiently compute the steady state of the original system with arbitrary precision.

\section{The dt-SHS Model}

This section formalizes the dt-SHS model first mentioned in section 1 . The mathematical framework is inspired by that in [1], but we model the presence of a physical forcing guard set rather than introducing state-dependent transition probabilities. The use of a discrete time framework is motivated by the simplicity in dealing with measurability issues for events on the underlying probability space, as well as by the direct computability of transition probabilities.

Definition 1 (dt-SHS). A discrete time stochastic hybrid system is a tuple $\mathcal{H}=\left(\mathcal{Q}, \mathcal{S}^{*}, \mathcal{G}, T, R\right)$, where 
- $\mathcal{Q}:=\left\{q_{1}, q_{2}, \ldots, q_{m}\right\}$, for some finite $m \in \mathbb{N}$, is the discrete component of the state space;

- $\mathcal{S}^{*}:=\cup_{i \in \mathcal{Q}}\{i\} \times \mathcal{D}_{i}^{*}$, is the hybrid state space, made up by a set of continuous "domains" for each mode $i \in \mathcal{Q}$, each of which is defined to be a compact subset $\mathcal{D}_{i}^{*} \subset \mathbb{R}^{n(i)}$. The function $n: \mathcal{Q} \rightarrow \mathbb{N}$ assigns to each $i \in \mathcal{Q}$ the dimension of the continuous state space $\mathbb{R}^{n(i)}$;

$-\mathcal{G}:=\cup_{i \in \mathcal{Q}}\{i\} \times \mathcal{G}_{i}, \mathcal{G}_{i}=\left\{g_{i j} ; j \in \mathcal{Q}, j \neq i, g_{i j} \subseteq \mathcal{D}_{i}^{*}\right\}$ is the set of spatial guards. We assume that $\forall i, j, k \in \mathcal{Q}, i \neq j \neq k, g_{i j} \cap g_{i k}=\varnothing$, and that the guards have non-trivial volume: $\mathcal{L}\left(g_{i j}\right) \neq 0, \forall i, j \in \mathcal{Q}, j \neq i$, where $\mathcal{L}(A)$ denotes the Lebesgue measure associated to any Borel subset $A \subset \mathcal{B}\left(\mathcal{D}_{i}^{*}\right)$. Let us further introduce the set $\mathcal{D}_{i}:=\mathcal{D}_{i}^{*} \backslash\left\{\cup_{\substack{j \in \mathcal{Q} \\ j \neq i}} g_{i j}\right\}$, the "invariant" of mode $i$, and $\mathcal{S}:=\cup_{i \in \mathcal{Q}}\{i\} \times \mathcal{D}_{i}$;

$-T: \mathcal{B}\left(\mathcal{D}_{(\cdot)}^{*}\right) \times \mathcal{S} \rightarrow[0,1]$ is a Borel-measurable stochastic kernel (the "transition kernel") on $\mathcal{D}_{(\cdot)}^{*}$ given $\mathcal{S}$, which assigns to each $s=(q, x) \in \mathcal{S}$ a probability measure on the Borel space $\left(\mathcal{D}_{q}^{*}, \mathcal{B}\left(\mathcal{D}_{q}^{*}\right)\right): T(d x \mid(q, x))$;

- $R: \mathcal{B}\left(\mathcal{D}_{(\cdot)}^{*}\right) \times \mathcal{G} \times \mathcal{Q} \rightarrow[0,1]$ is a Borel-measurable stochastic kernel (the "reset kernel") on $\mathcal{D}_{(\cdot)}^{*}$, given $\mathcal{G} \times \mathcal{Q}$, that assigns to each $s=(q, x) \in \mathcal{G}$, and $q^{\prime} \in \mathcal{Q}, q^{\prime} \neq q$, a probability measure on the Borel space $\left(\mathcal{D}_{\left(q^{\prime}\right)}^{*}, \mathcal{B}\left(\mathcal{D}_{\left(q^{\prime}\right)}^{*}\right)\right)$ : $R\left(d x \mid(q, x), q^{\prime}\right)$.

The system initialization at the initial time (say $k=0$ ) is specified by some probability measure $\pi_{0}: \mathcal{B}\left(\mathcal{S}^{*}\right) \rightarrow[0,1]$ on the Borel space $\left(\mathcal{S}^{*}, \mathcal{B}\left(\mathcal{S}^{*}\right)\right)$. Here again $\mathcal{B}\left(\mathcal{S}^{*}\right)$ is the $\sigma$-field generated by the subsets of $\mathcal{S}^{*}$ of the form $\cup_{q}\{q\} \times B_{q}$, with $B_{q}$ denoting a Borel set in $\mathcal{D}_{q}^{*}$. For details on the measurability and metric properties of $\mathcal{H}$, the reader is invited to refer to $[1,5]$. Notice that the transition and reset kernels (respectively $T$ and $R$ ) have different domains of definition $(\mathcal{S}$ and $\mathcal{G} \times \mathcal{Q}$ ), but the same support $\left(\mathcal{D}^{*}\right)$. Next, we define the notion of execution for the above model (throughout the paper, random processes will be denoted in bold fonts, while random variables in normal typesets).

Definition 2 (Execution). Consider a dt-SHS $\mathcal{H}=(\mathcal{Q}, n, \mathcal{G}, T, R)$. An execution for $\mathcal{H}$, associated with an initial distribution $\pi_{0}$, is a stochastic process $\{\mathbf{s}(k), k \in[0, N], N \in \mathbb{N}\}$ with values in $\mathcal{S}^{*}$, whose sample paths are obtained according to the following algorithm:

extract from $\mathcal{S}^{*}$ a value $s_{0}=\left(q_{0}, x_{0}\right)$ for $\mathbf{s}(0)$, according to the distribution $\pi_{0}$;

for $k=0$ to $N-1$,

if there is a $j \neq q_{k}, j \in \mathcal{Q}$, such that $x_{k} \in g_{q_{k}, j}$,

then extract a value $s_{k+1} \in \mathcal{S}^{*}$ for $\mathbf{s}(k+1)$, according to $R\left(\cdot \mid s_{k}, j\right)$;

else extract a value $s_{k+1} \in \mathcal{S}^{*}$ for $\mathbf{s}(k+1)$, according to $T\left(\cdot \mid s_{k}\right)$;

end.

As mentioned, the introduced (autonomous) dt-SHS is related to the (controlled) SHS in [1], where the additional presence of a stochastic kernel allows for the presence of spontaneous jumps within the invariants. The theory developed in this work can be extended to account for similar terms. 


\section{Markov set-chains}

We define here the concept of Markov set-chain, which will be used as an abstraction framework for dt-SHS. We also recall some useful results from [8], which contains a compendium of literature on the subject.

Definition 3 (Transition Set). Let $P, Q \in \mathbb{R}^{n \times n}$, with $P, Q \geq 0$ (that is component-wise nonnegative matrices, not necessarily stochastic), with $P \leq Q$. We define a "transition set" as:

$$
[P, Q]=\left\{A \in \mathbb{R}^{n \times n}: A \text { is a stochastic matrix and } P \leq A \leq Q\right\} .
$$

In the proceeding, we assume that the transition set $[P, Q] \neq \varnothing$. When the "bounding matrices" $P, Q$ will be clear from the context, we will use the notation $[\Pi]$ to denote such compact (possibly infinite) set of stochastic matrices. We can define a Markov set-chain as a non-homogeneous, discrete-time Markov chain, where the transition probabilities vary non-deterministically within a compact transition set $[\Pi]$. More formally,

Definition 4 (Markov set-chain). Let $[\Pi]$ be a transition set, i.e. a compact set of $n \times n$ stochastic matrices. Consider the set of all non-homogeneous Markov chains having all their transition matrices in $[\Pi]$. We call the sequence

$$
[\Pi],[\Pi]^{2}, \cdots
$$

a Markov set-chain, where $[\Pi]^{k}$ is defined by induction as the compact set of all possible products $A_{1}, \cdots, A_{k}$, such that, $\forall i=1, \cdots, k, A_{i} \in[\Pi]$.

Similarly, let $\left[\pi_{0}\right]$ be a compact set of $1 \times n$ stochastic vectors, introduced as in Def. 3. We call $\left[\pi_{0}\right]$ the initial distribution set.

The compact set $\left[\pi_{k}\right]=\left[\pi_{0}\right][\Pi]^{k}$ is the $k$-th distribution set and

$$
\left[\pi_{0}\right],\left[\pi_{0}\right][\Pi], \cdots
$$

is the Markov set-chain with initial distribution set $\left[\pi_{0}\right]$.

It can be shown that each element $\left[\pi_{k}\right]$ is a convex polytope if $\left[\pi_{0}\right]$ is a convex polytope and $[\Pi]$ is a transition set. It should be noticed that the number of vertices of $\left[\pi_{k}\right]$ increases with $k$, thus the computational burden to obtain $\left[\pi_{k}\right]$ for large values of $k$ should be accounted for. However, it is possible to compute tight (see [8]) upper and lower bounding matrices $L_{k}, H_{k}$ for $\left[\pi_{k}\right]$ in a very efficient way, in particular the computation of $L_{k}, H_{k}$ can be recursively obtained from $L_{k-1}, H_{k-1}$.

Definition 5 (Coefficient of Ergodicity). For any stochastic matrix A, its coefficient of ergodicity is defined as follows:

$$
\mathcal{T}(A)=\frac{1}{2} \max _{i, j}\left\|a_{i}-a_{j}\right\|,
$$

where $a_{i}$ is the $i$-th row of $A$ and $\|\cdot\|$ on a vector is the standard 1-norm. If $\mathcal{T}(A)<1, A$ is said to be scrambling. 
The above definition can be directly extended to Markov set-chains:

Definition 6. For any transition set $[\Pi]$, its coefficient of ergodicity is defined as follows:

$$
\mathcal{T}([\Pi])=\max _{A \in[\Pi]} \mathcal{T}(A) .
$$

Notice that since $\mathcal{T}(\cdot)$ is a continuous function and $[\Pi]$ a compact set, the maximum argument of $\mathcal{T}([\Pi])$ exists. Also notice that $\mathcal{T}([\Pi]) \in[0,1]$, as $\mathcal{T}(A) \in[0,1]$. This value provides a measure of the "contractive" nature of the Markov setchain: the smaller $\mathcal{T}([\Pi])$, the more contractive the MSC. This will become clear when studying the asymptotic properties of the MSC, and is related to the regularity properties of the matrices that build up the MSC [8]. The exact value of $\mathcal{T}([\Pi])$ can be hard to compute, but it can be upper bounded as follows:

Theorem 1. Let $[\Pi]$ be the interval $[P, Q]$ and $A \in[\Pi]$, then:

$$
|\mathcal{T}([\Pi])-\mathcal{T}(A)| \leq\|Q-P\|
$$

The above matrix norm is taken from [8] and is a modification of the induced 1-norm. The following notion connects to Definition 5 :

Definition 7 (Scrambling Integer). Suppose $r \geq 1$ is such that $\mathcal{T}\left(A_{1} \cdots A_{r}\right)<$ $1, \forall A_{1}, \cdots, A_{r} \in[\Pi]$. Then $[\Pi]$ is said to be product scrambling and $r$ its scrambling integer.

We now illustrate some results on the convergence of MSC.

Theorem 2. Given a product scrambling $M S C$ with transition set $[\Pi]$ and initial distribution set $\left[\pi_{0}\right]$, then there exists a unique limit set $\left[\pi_{\infty}\right]$ such that $\left[\pi_{\infty}\right][\Pi]=\left[\pi_{\infty}\right]$. Moreover, let $r$ be the scrambling integer of $[\Pi]$. Then for any positive integer $h$, and according to the Hausdorff metric $d(\cdot)$ on compact sets:

$$
d\left(\left[\pi_{h}\right],\left[\pi_{\infty}\right]\right) \leq K \beta^{h}
$$

where $K=\left[\mathcal{T}\left([\Pi]^{r}\right)\right]^{-1} d\left(\left[\pi_{0}\right],\left[\pi_{\infty}\right]\right)$ and $\beta=\mathcal{T}\left([\Pi]^{r}\right)^{\frac{1}{r}}<1$. Thus

$$
\lim _{h \rightarrow \infty}\left[\pi_{h}\right]=\lim _{h \rightarrow \infty}\left[\pi_{0}\right][\Pi]^{h}=\left[\pi_{\infty}\right] .
$$

As we argued before, the exact computation of $\left[\pi_{\infty}\right]$ can be expensive. However, it is possible to use the upper and lower bounding matrices $L_{k}, H_{k}$ mentioned above to obtain an accurate estimate of $\left[\pi_{\infty}\right]$ with a reasonable computational complexity. In fact, $L_{k}, H_{k}$ converge to a value $L_{\infty}, H_{\infty}$ such that $\left[\pi_{\infty}\right] \subseteq\left[L_{\infty}, H_{\infty}\right]$. Define the diameter of a compact set (referred to either matrices or vectors) as

$$
\Delta([\Pi])=\max _{A, A^{\prime} \in[\Pi]}\left\|A-A^{\prime}\right\| .
$$

The following result provides an efficient procedure to compute an upper bound for the diameter of the limit set $\left[\pi_{\infty}\right]$. 
Theorem 3. Given a product scrambling Markov set-chain with transition set $[\Pi]=[P, Q]$ and such that $\mathcal{T}([\Pi])<1$, then

$$
\Delta\left(\left[\pi_{\infty}\right]\right) \leq \frac{\Delta([\Pi])}{1-\mathcal{T}([\Pi])} \leq \frac{\|Q-P\|}{1-\mathcal{T}(A)-\|Q-P\|},
$$

for any $A \in[\Pi]$. The second inequality holds only if $\mathcal{T}(A)+\|Q-P\| \leq 1$.

\section{Probabilistic Dynamics}

The model described in Definition 1 is quite general and allows for a wealth of possible behaviors. However, even in the case of further knowledge of the structure of the dynamics (beyond the general stochastic kernels $T, R$ that characterize it), is in general not translatable into a closed-form expression for the solution process of $\mathcal{H}$. Thus, in order to study the dynamical properties of $\mathcal{H}$, two directions can be pursued. The first looks at the ensemble of possible realizations that originate from the initial distribution, according to the steps in Definition 2. Monte Carlo simulations are a known example of this approach. The second, instead, characterizes probabilistically the presence of the solution process in certain regions of $\mathcal{S}^{*}$, as time progresses. More precisely, it is of interest to define the following likelihood: given a point $s_{0} \in \mathcal{S}^{*}$, what is the probability that the solution process $\mathbf{s}(\cdot)$ of $\mathcal{H}$, starting from $s_{0}$, is located in the set $A \in \mathcal{B}\left(\mathcal{S}^{*}\right)$ at time $k>0$ ? Similarly, given a point $s_{0} \in \mathcal{S}^{*}$, what is the probability that the solution process $\mathbf{s}(\cdot)$ of $\mathcal{H}$ stays within the set $A \in \mathcal{B}\left(\mathcal{S}^{*}\right)$, if $s_{0} \in A$, for all the time $k \in[0, N], N<\infty$ ?

These and similar quantities leverage the ability of defining and computing the concept of probabilistic reachability [1]. Interestingly, these stochastic reachability problems are related to the two analogous deterministic approaches taken in [6] for constructing finite abstractions of (deterministic) HS. The two probabilistic kernels $T$ and $R$ depend on, respectively, the invariant and the guard sets. We are thus particularly interested in computing the transition probabilities between these subsets of the hybrid state space. For instance, considering two modes $q, q^{\prime} \in \mathcal{Q}$, we call $p_{q, q^{\prime}}(x)$ the probability that a trajectory, starting from a point $(q, x) \in \mathcal{S}$, has to transition in a time step (according to $T(\cdot \mid(q, x)))$ into any other domain $q^{\prime} \neq q$ by intersecting the corresponding guard, or possibly to continue evolving in $q^{\prime}=q$ :

$$
\begin{aligned}
p_{q, q^{\prime}}(x) & \triangleq \int_{g_{q, q^{\prime}}} T(d y \mid(q, x)), \text { if } q^{\prime} \neq q, \\
p_{q, q}(x) & \triangleq \int_{\mathcal{D}_{q}} T(d y \mid(q, x))=1-\sum_{\substack{q^{\prime} \in \mathcal{Q} \\
q^{\prime} \neq q}} \int_{g_{q, q^{\prime}}} T(d y \mid(q, x)) .
\end{aligned}
$$

The case where $(q, x) \in \mathcal{S}^{*} \backslash \mathcal{S}$, which is associated to the probability that the trajectory is reset, according to $R\left(\cdot \mid(q, x), q^{\prime}\right)$, into an invariant $q^{\prime} \neq q$, is similar. 
Let us denote this probability $p_{\left(q, q^{\prime}\right), q^{\prime}}(x)$ :

$$
p_{\left(q, q^{\prime}\right), q^{\prime}}(x) \triangleq \int_{\mathcal{D}_{q^{\prime}}} R\left(d y \mid(q, x), q^{\prime}\right) .
$$

Notice that, as the support of $T$ and of $R$ coincides, the contribution of both terms is similar, except for the fact that $T$ is associated with a one time-step continuous motion, while $R$ to an instantaneous reset.

Investigating similar quantities for dynamics over a longer time interval involves conditioning the probability backwards in time and referring to the "template quantities" discussed above. For instance, we may be interested in the following transition, for $q, r, s \in \mathcal{Q}, q \neq r, r \neq s: x \in g_{q, r} \stackrel{R}{\rightarrow} \mathcal{D}_{r} \stackrel{T}{\rightarrow} g_{r, s} ;$ and the associated probability $p_{(q, r), r}(x) p_{r, s}(\cdot)$. This is computed by:

$$
\begin{aligned}
\mathcal{P}\left(\mathbf{s}(1) \in g_{r, s} \mid \mathbf{s}(0)=(q, x) \in g_{q, r}\right) & =\int_{\mathcal{D}_{r}} \int_{g_{r, s}} R(d y \mid(q, x), r) T(d z \mid(r, y)) \\
& =\int_{\mathcal{D}_{r}} R(d y \mid(q, x), r) p_{r, s}(y)
\end{aligned}
$$

This quantity shows that the contributions of the one-step probabilities over time have to be necessarily "averaged" over the influence of the stochastic kernels that precede them. This will also hold with reference to a particular initial distribution $\pi_{0}$. As already mentioned, the interplay between transition and reset probabilities is a characteristic feature of SHS.

The terms in (2)-(3), and their multiplications, are then characteristic of the computations we want to perform to study the dynamics of the dt-SHS $\mathcal{H}$. In principle, we may be able to associate a transition probability to each couple of elements taken from the set of invariants and guards. This would allow to abstract the dynamics of $\mathcal{H}$ into those of a discrete $m^{2}$-dimensional MC (where $m=\operatorname{card}(\mathcal{Q}))$. However, by closely looking at the quantity in (2) [resp. (3)], it becomes clear that it is necessary to compute the transition probabilities over the whole invariant $\mathcal{D}_{q}$ [over the whole guard $g_{q, q^{\prime}}$, averaged over the contribution of the incoming reset maps $R(\cdot \mid(\cdot, \cdot), q)$ [the transition kernel $T(\cdot \mid(q, \cdot))]$. To fully make sense, these last quantities would have to depend on other probabilities, and so on backwards, until integrating over an initial distribution. This computation is rather unfeasible, and its bottleneck hinges on the dependence of $T$ and $R$ on the continuous component of the hybrid state space.

Rather than aiming, as just proposed, at abstracting the dynamics of the dtSHS $\mathcal{H}$ into an $m^{2}$-dimensional MC, we may instead allow an abstraction into a higher dimensional structure, while improving the precision of the approximation. The technique to achieve this, described in the following section, is based on a continuity assumption on the dynamics, and a state-partitioning procedure.

\section{Abstraction Procedure}

This section describes the abstraction procedure for the dt-SHS model $\mathcal{H}$ of section 2. The dt-SHS $\mathcal{H}$ will be abstracted into a Markov set-chain $\mathcal{M}$, described 
by a one-step transition set $[\Pi]=[P, Q]$. The computations involved in obtaining the abstraction are reduced to integrations over the continuous part of the hybrid state space. The procedure introduces some necessary approximations in order to perform the computations feasibly. However, explicit bounds on these errors will be obtained, provided some continuity assumptions are raised. The association of these bounds to the computed transition probabilities allows a connection with the theory of MSC, as it provides a direct definition of the transition set $[\Pi]$ of $\mathcal{M}$. The precision of the abstraction will depend on a parameter $\delta$. It is desirable for the abstraction to be endowed, in the limit as $\delta \rightarrow 0$, with some convergence properties to the original dt-SHS $\mathcal{H}$.

\section{Approximation of state-dependent Transitions and Resets}

As discussed in section 4, the dependence of transition and reset kernels on, respectively, the invariant and the guard set, and their continuous supports, renders the computation of transition probabilities via nested integrals of product terms as in (4) computationally unattractive. Introducing some "regularity assumptions" on the probabilistic kernels, it is possible to achieve a "statememoryless" approximation for these transition probabilities, whereby their calculation does not depend on the continuous part of the hybrid state-space $\mathcal{S}$.

Let us suppose that the stochastic kernels $T$ and $R$, which depend on the continuous component of the hybrid state in Definition 1 of $\mathcal{H}$, admit densities respectively $t$ and $r$. Similarly, let us assume the initial probability distribution $\pi_{0}$ has a density $p_{0}$. It is supposed that $p_{0}, t$, and $r$ satisfy the following Lipschitz condition.

\section{Assumption 1 (Lipschitz Continuity of the Stochastic Kernels)}

1. $\left|p_{0}(s)-p_{0}\left(s^{\prime}\right)\right| \leq k_{0}\left\|x-x^{\prime}\right\|$, for all $s=(q, x), s^{\prime}=\left(q, x^{\prime}\right) \in \mathcal{D}_{q}^{*}$;

2. $\left|t(\bar{x} \mid s)-t\left(\bar{x} \mid s^{\prime}\right)\right| \leq k_{T}\left\|x-x^{\prime}\right\|$, for all $s=(q, x), s^{\prime}=\left(q, x^{\prime}\right) \in \mathcal{D}_{q}$, and $(q, \bar{x}) \in \mathcal{D}_{q}^{*}$

3. $\left|r(\bar{x} \mid s, \bar{q})-r\left(\bar{x} \mid s^{\prime}, \bar{q}\right)\right| \leq k_{R}\left\|x-x^{\prime}\right\|$, for all $s=(q, x), s^{\prime}=\left(q, x^{\prime}\right) \in \mathcal{D}_{q}^{*} \backslash \mathcal{D}_{q}$, $(\bar{q}, \bar{x}) \in \mathcal{D}_{\bar{q}}^{*}$, and $\bar{q} \in \mathcal{Q}, \bar{q} \neq q$,

where $k_{0}, k_{T}$, and $k_{R}$ are finite positive constants.

Let us also recall the implicit assumption, raised for computations' sake in Definition 1 , that for each $q \in \mathcal{Q}$, the continuous domain $\mathcal{D}_{q}^{*}$ associated to such mode is a bounded subset of $\mathbb{R}^{n(q)}$.

Let us introduce the following quantities (see Table 1 for a compendium of them), describing the (finite) volume measures of particular subsets of the domains: $\lambda_{q}^{*}=\mathcal{L}\left(\mathcal{D}_{q}^{*}\right), \lambda_{q}=\mathcal{L}\left(\mathcal{D}_{q}\right), \lambda_{q, r}=\mathcal{L}\left(g_{q, r}\right), \lambda=\sum_{q \in \mathcal{Q}} \mathcal{L}\left(D_{q}\right), \lambda^{*}=\sum_{q \in \mathcal{Q}} \mathcal{L}\left(D_{q}^{*}\right)$, where $\mathcal{L}$ is the Lebesgue measure of a bounded subset of a Euclidean space. Since $\mathcal{D}_{q}^{*}=\mathcal{D}_{q} \cup \mathcal{G}_{q}$, it follows that $\forall q \in \mathcal{Q}, \lambda_{q}=\lambda_{q}^{*}-\sum_{\substack{r \in \mathcal{Q} \\ r \neq q}} \lambda_{q, r}$.

Let us now focus on the computation of the transition probabilities. Consider a 
mode $q \in \mathcal{Q}$, and any two points $(q, x),\left(q, x^{\prime}\right) \in \mathcal{D}_{q}$. Then, with reference to the quantity in (2) and according to Assumption 1, let us compute, $\forall r \in \mathcal{Q}, r \neq q$,

$$
\begin{array}{r}
\left|p_{q, r}(x)-p_{q, r}\left(x^{\prime}\right)\right|=\left|\int_{g_{q, r}} T(d z \mid(q, x))-\int_{g_{q, r}} T\left(d z \mid\left(q, x^{\prime}\right)\right)\right| \\
\leq \int_{g_{q, r}}\left|T(d z \mid(q, x))-T\left(d z \mid\left(q, x^{\prime}\right)\right)\right| \leq \lambda_{q, r} k_{T}\left\|x-x^{\prime}\right\| .
\end{array}
$$

A similar bound is obtained for the case $r=q$, which now depends on the quantity $\lambda_{q}$. Furthermore, a similar bound can be found for the quantity in (3): selecting any two points $(q, x),\left(q, x^{\prime}\right) \in g_{q, r} \subset \mathcal{D}_{q}^{*}, r \neq q$, we have:

$$
\begin{aligned}
\mid p_{(q, r), r}(x)- & p_{(q, r), r}\left(x^{\prime}\right)|=| \int_{\mathcal{D}_{r}} R(d z \mid(q, x), r)-\int_{\mathcal{D}_{r}} R\left(d z \mid\left(q, x^{\prime}\right), r\right) \mid \\
& \leq \int_{\mathcal{D}_{r}}\left|R(d z \mid(q, x), r)-R\left(d z \mid\left(q, x^{\prime}\right), r\right)\right| \leq \lambda_{q} k_{R}\left\|x-x^{\prime}\right\| .
\end{aligned}
$$

Likewise, it is possible to derive error bounds for more complicated expressions, such as (4).

\section{Hybrid State Space Partition}

Let us now introduce a partition of the hybrid state space $\mathcal{S}^{*}$ (see Table 1 ). Recall that $\mathcal{S}^{*}$ can be written as $\mathcal{S}^{*}=\cup_{q \in \mathcal{Q}}\{q\} \times \mathcal{D}_{q}^{*}=\cup_{q \in \mathcal{Q}}\{q\} \times\left\{\underset{\substack{r \in \mathcal{Q} \\ r \neq q}}{ } g_{q, r} \cup \mathcal{D}_{q}\right\}$.

With regards to a particular mode $q \in \mathcal{Q}$, let us introduce a partition of $\mathcal{D}_{q}^{*}$ of cardinality $c_{q}^{\delta}=d_{q}^{\delta}+\sum_{\substack{r \in \mathcal{Q} \\ r \neq q}} e_{q, r}^{\delta}$, where the first term $d_{q}^{\delta}$ refers to the number of sections of the invariant $\mathcal{D}_{q}$, while the other terms $e_{q, r}^{\delta}$ refer to the cardinality of the partition of the corresponding guard set $g_{q, r}$. These terms are clearly all greater than or equal to one. Let us introduce their respective measures $\lambda_{q}^{j}$ and $\lambda_{q, r}^{k}$, which make up the quantities $\lambda_{q}=\sum_{j=1}^{d_{q}^{\delta}} \lambda_{q}^{j}$, and $\lambda_{q, r}=\sum_{k=1}^{e_{q, r}^{\delta}} \lambda_{q, r}^{k}$ introduced above. This dependence on the parameter $\delta$ will be made clear shortly. We do not impose any structure on the partition, but only require it, within each domain, to respect (that is, not to intersect) the boundaries between invariant and guards, and those between each couple of adjacent guards. It is then possible to express the domain $\mathcal{D}_{q}^{*}$, associated to mode $q \in \mathcal{Q}$, as the union of the following disjoint sets:

$$
\mathcal{D}_{q}^{*}=\left\{\cup_{\substack{r \in \mathcal{Q} \\ r \neq q}}\left\{\cup_{j=1}^{e_{q, r}^{\delta}} g_{q, r}^{j}\right\}\right\} \cup\left\{\cup_{j=1}^{d_{q}^{\delta}} \mathcal{D}_{q}^{j}\right\} .
$$

Let us now associate a discrete state of $\mathcal{M}$ to each of these partitions, by introducing mode $q^{j}$ for $\mathcal{D}_{q}^{j}$, and mode $q_{r}^{j}$ for $g_{q, r}^{j}$. The parameter $\delta$ is defined to be

$$
\begin{gathered}
\delta=\max _{q \in \mathcal{Q}}\left\{\max \left\{\epsilon_{q}, \gamma_{q}\right\}\right\}, \text { where } \\
\epsilon_{q}=\max _{\substack{r \in \mathcal{Q} \\
r \neq q}} \operatorname{lil}_{j, \ldots, e_{q, r}^{\delta}} \sup \left\{\left\|x-x^{\prime}\right\|: x, x^{\prime} \in g_{q, r}^{j}\right\}=\max _{\substack{r \in \mathcal{Q} \\
r \neq q}, 1, \ldots, e_{q, r}^{\delta}} \epsilon_{q, r}^{j}, \\
\gamma_{q}=\max _{j=1, \ldots, d_{q}^{\delta}} \sup \left\{\left\|x-x^{\prime}\right\|: x, x^{\prime} \in \mathcal{D}_{q}^{j}\right\}=\max _{j=1, \ldots, d_{q}^{\delta}} \gamma_{q}^{j} .
\end{gathered}
$$


In other words, $\delta$ represents the largest diameter of the partitions defined on $\mathcal{S}^{*}$. Let us choose a representative point within each single mode introduced through the partition: $\forall q \in \mathcal{Q}, \forall j=1, \ldots, d_{q}^{\delta}$, let us select a point $\bar{x}_{q}^{j} \in \mathcal{D}_{q}^{j} ; \forall q \in \mathcal{Q}, r \neq$ $q, \forall j=1, \ldots, e_{q, r}^{\delta}$, let us select a point $\bar{x}_{q, r}^{j} \in g_{q, r}^{j}$.

We will now revisit the computation of the probabilistic quantities in (2)-(3) with the additional knowledge of the derived error bounds, in order to define the elements of the MSC $\mathcal{M}$ that abstracts the original dt-SHS $\mathcal{H}$. Toward this aim, we associate to each discrete state of the above partition a distinct state of the MSC. The values of the error bounds depend on the partition diameter $\delta$, and on the structure of the dynamics of $\mathcal{H}$. To be more precise, we shall approximate the quantities in (2)-(3) with ones that will be based on computations performed on the representative points. The new transition probabilities will be intuitively denoted in a similar fashion as the relations in (2)-(3). Let us start from the relation in (2):

$$
\forall x \in \mathcal{D}_{q}^{j}, p_{q^{j}, r^{k}}(x)=\int_{g_{q, r}^{k}} T(d y \mid(q, x)) \approx p_{q^{j}, r^{k}}\left(\bar{x}_{q}^{j}\right),
$$

More precisely, $\left|p_{q^{j}, q_{r}^{k}}(x)-p_{q^{j}, q_{r}^{k}}\left(\bar{x}_{q}^{j}\right)\right| \leq \lambda_{q, r}^{k} k_{T} \gamma_{q}^{j} \leq \lambda^{*} k_{T} \delta$. Notice that, if $x \in$ $\mathcal{D}_{q}^{j} \subseteq \mathcal{D}_{q}, T(d y \mid(q, x))=T\left(d y \mid\left(q^{j}, x\right)\right)$.

Now, focusing on equation (3), we have:

$$
\forall x \in g_{q, r}^{j}, p_{(q, r)^{j}, r^{k}}(x)=\int_{\mathcal{D}_{r}^{k}} R(d y \mid(q, x), r) \approx p_{(q, r)^{j}, r^{k}}\left(\bar{x}_{q, r}^{j}\right),
$$

where $\left|p_{(q, r)^{j}, r^{k}}(x)-p_{(q, r)^{j}, r^{k}}\left(\bar{x}_{q, r}^{j}\right)\right| \leq \lambda_{r}^{k} k_{R} \epsilon_{q, r}^{j} \leq \lambda^{*} k_{R} \delta$. Notice that, if $x \in$ $g_{q, r}^{j} \subseteq \mathcal{D}_{q}^{*}, R(d y \mid(q, x), r)=T\left(d y \mid\left(g_{q, r}^{j}, x\right), D_{r}^{k}\right)$.

Similar transition probabilities and bounds can be referred to the initial distribution $\pi_{0}$. Moreover, it is possible to compute analogous bounds for quantities, such as (4), which involve more than a single step of computation. However, it will become clear in the next section that these bounds can be equivalently derived from direct matrix computations on the $\operatorname{MSC} \mathcal{M}$.

\section{Steady state computation using the MSC abstraction}

In this section we show that it is possible to infer the asymptotic behavior of the dt-SHS $\mathcal{H}$ using the introduced Markov set-chain abstraction $\mathcal{M}$. We start by providing an intuitive justification of why the MSC $\mathcal{M}$ may yield some conclusions about the asymptotic dynamics of the original dt-SHS $\mathcal{H}$.

The values of the MSC, i.e. the explicit bounds for the errors associated to the approximate computations of the transition probabilities of the dt-SHS, allow to introduce a "conservative estimate" of the actual transition probabilities between regions of the state space of the original dt-SHS. By selecting a small enough diameter $\delta$ of the partition, the possibly contractive nature of $\mathcal{M}$ may dominate over the approximation errors. The contractivity of $\mathcal{M}$ depends on the dynamics 


\begin{tabular}{|c|c|c|c|c|c|c|}
\hline component & form & parts & partitions & cardinality & size & diameter \\
\hline \hline $\begin{array}{c}\text { hybrid space } \\
\text { of } \mathcal{H}\end{array}$ & $\mathcal{S}^{*}=\bigcup_{q \in \mathcal{Q}}\{q\} \times \mathcal{D}_{q}^{*}$ & $\mathcal{D}_{q}^{*}$ & & $\mathfrak{c}^{\delta}$ & $\lambda^{*}=\sum_{q \in \mathcal{Q}} \lambda_{q}^{*}$ & $\delta$ \\
\hline domain & $\mathcal{D}_{q}^{*}=\mathcal{D}_{q} \cup \mathcal{G}_{q}$ & $\mathcal{D}_{q}, \mathcal{G}_{q}$ & & $c_{q}^{\delta}$ & $\lambda_{q}^{*}$ & $\gamma_{q} \vee \epsilon_{q}$ \\
\hline invariant & $\mathcal{D}_{q}$ & & $\mathcal{D}_{q}^{j}$ & $d_{q}^{\delta}$ & $\lambda_{q}$ & $\gamma_{q}$ \\
\hline $\begin{array}{c}\text { invariant } \\
\text { sections }\end{array}$ & $\mathcal{D}_{q}^{j}$ & & & 1 & $\lambda_{q}^{j}$ & $\gamma_{q}^{j}$ \\
\hline guards & $\mathcal{G}_{q}=\bigcup_{\substack{r \in \mathcal{Q} \\
r \neq q}}\left\{g_{q, r}\right\}$ & $g_{q, r}$ & $g_{q, r}^{j}$ & $\sum_{\substack{r \in \mathcal{Q} \\
r \neq q}} e_{q, r}^{\delta}$ & $\lambda_{q}^{*}-\lambda_{q}$ & $\epsilon_{q}$ \\
\hline $\begin{array}{c}\text { guard } \\
\text { sections }\end{array}$ & $g_{q, r}^{j}$ & & & 1 & $\lambda_{q, r}^{j}$ & $\epsilon_{q, r}^{j}$ \\
\hline
\end{tabular}

Table 1. Relationship between the components of the dt-SHS and the elements of the partition that yields the MSC, with corresponding quantities of interest.

and on the structure of $\mathcal{H}$. By tuning the parameter $\delta$, we may derive conclusions on the asymptotic behavior of $\mathcal{H}$.

We now make the above discussion more quantitative. Given a desired precision $\varepsilon>0$ on the approximation, we integrate the procedure for the partition of $\mathcal{H}$ into an algorithm to compute the steady state of the MSC abstraction $\mathcal{M}$. The precision $\varepsilon$ is related to the partition parameter $\delta$. As discussed above, the steady state vector $\left[\pi_{\infty}\right]$ for $\mathcal{M}$ is an estimate of the invariant measure of $\mathcal{H}$, with a confidence bound given by the diameter $\Delta\left(\left[\pi_{\infty}\right]\right)$. Let us initialize a partition of $\mathcal{H}$ according to a value $\delta=\delta(\varepsilon)$, which guarantees a precision $\varepsilon$ for the steady state computation of $\left[\pi_{\infty}\right]$. The transition set $[\Pi]=[P, Q]$, as constructed in the previous section, has the following property:

$$
\Delta([\Pi]) \leq\|Q-P\|=\lambda^{*} \bar{k} \delta(\varepsilon),
$$

with $\bar{k}=k_{T} \vee k_{R} \vee k_{0}$. The inequality can be drawn by directly employing the definition of matrix norm, and the bounds derived for equations (5)-(6). Then, by Theorem 3 , a sufficient condition to achieve $\Delta\left(\left[\pi_{\infty}\right]\right) \leq \varepsilon$ is the following:

$$
\lambda^{*} \bar{k} \delta(\varepsilon) \leq \varepsilon(1-\mathcal{T}([\Pi])) .
$$

It is clear that if $\mathcal{T}([\Pi])<1$ there always exists a value of $\delta(\varepsilon)$ that satisfies this inequality, since the LHS expression goes to zero as $\delta(\varepsilon)$ goes to zero. Notice however that, without an idea of the transition probabilities that define $[\Pi]$, one cannot estimate $\mathcal{T}([\Pi])$. Since in general $0 \leq \mathcal{T}([\Pi]) \leq 1$, the set of feasible values for $\delta(\varepsilon)$ that satisfy equation (8) ranges from a finite upper bound $\delta_{0}$ (when $\mathcal{T}([\Pi])=0$ ) to 0 (when $\mathcal{T}([\Pi])=1$ ). This makes sense: until we have no information about the contractive nature of $[\Pi]$, there is no possibility to estimate the limit set behavior. For this reason, it is impossible to establish a priori a value for $\delta(\varepsilon)$ that guarantees a desired precision $\varepsilon$ in the steady state computation using the abstraction $\mathcal{M}$.

However, we can choose an "optimistic" initial value $\delta_{0}$. In the following iterative algorithm, given a partition diameter $\beta(k)$, we define $\Pi(k)$ the transi- 
tion probabilities computed by the abstraction algorithm described in the previous section from an initial distribution $\pi_{0}$. Moreover, we define $[\Pi(k)]=$ $[P(k), Q(k)]$, the associated MSC. The parameter $\alpha(k)$ represents an upper bound for $\mathcal{T}([\Pi(k)])$.

\section{Algorithm 1 (Compute steady state of $\mathcal{H}$ with precision $\varepsilon$ )}

input: $(\mathcal{H}, \varepsilon)$;

set integer $k=0$, real $\alpha(0)=0$, and real $\beta(0)$ such that $\lambda^{*} \bar{k} \beta(0) \leq \varepsilon$;

for $k \geq 0$

compute $\Pi(k),[\Pi(k)]=[P(k), Q(k)]$ according to $\beta(k) ;$

set $\alpha(k)=\mathcal{T}(\Pi(k))+\|Q(k)-P(k)\| ;$

if $\alpha(k) \geq 1$ or $\alpha(k) \geq \alpha(k-1)$ then set $\beta(k+1)=a \beta(k), a<1$;

else if $\frac{\|Q(k)-P(k)\|}{1-\alpha(k)}>\varepsilon$ then set $\beta(k+1)$ s.t. $\lambda^{*} \bar{k} \beta(k+1) \leq \varepsilon(1-\alpha(k))$;

else exit;

set $k=k+1$;

end

compute the steady state $\pi_{\infty}$ of $\Pi(k)$;

output: $\left(\pi_{\infty}\right)$.

Notice that, $\forall k \geq 0, \beta(k+1)<\beta(k)$, thus $\lim _{k \rightarrow+\infty} \Delta([\Pi])=\lim _{k \rightarrow+\infty} \| Q(k)-$ $P(k) \|=0$. Let $\mathcal{T}_{\infty}=\lim _{k \rightarrow+\infty} \mathcal{T}([\Pi(k)])<1$ : by Theorem 1 it follows that $\lim _{k \rightarrow+\infty} \alpha(k)=\mathcal{T}([\Pi(k)])=\mathcal{T}_{\infty}$. Namely, if $\mathcal{T}_{\infty}<1$ we can arbitrarily increase the accuracy $\delta$ of our abstraction until, by equation $(8), \lambda^{*} \bar{k} \delta \leq \varepsilon\left(1-\mathcal{T}_{\infty}\right)$. When this happens the algorithm terminates, and we compute the steady state $\pi_{\infty}$, or compute $\left[\pi_{\infty}\right]$ using the upper and lower bounding matrices $L_{\infty}, H_{\infty}$, as described in section 3 .

We now discuss the computational burden of our procedure. It is clear that the main bottlenecks are (1) the abstraction procedure for the partitioning of the hybrid state space; and (2) the limit set computation on the abstraction MSC. The first computation directly depends on the parameter $\delta$, which is related to $\mathcal{T}_{\infty}$ by $(8)$. The second computation depends on two parameters: the cardinality of the MSC and the convergence speed. The state cardinality is $\mathfrak{c}^{\delta}$, and depends on $\delta$, while the convergence speed can be related to $\mathcal{T}_{\infty}$ by (1). The main weight in the computational complexity of our abstraction procedure is $\mathcal{T}_{\infty}$.

For the above arguments, it can be interesting to interpret $\mathcal{T}_{\infty}$ as the coefficient of ergodicity $\mathcal{T}(\mathcal{H})$ of the dt-SHS $\mathcal{H}$, and possibly compare this value with other convergence bounds directly derived on the structure and the dynamics of $\mathcal{H}$. 


\section{Numerical Study}

We implement the proposed abstraction procedure on a simple one-dimensional dynamical system, whose dynamics is described by the following SDE, defined for $t \geq 0$ :

$$
d \mathbf{X}_{t}=f\left(\mathbf{X}_{t}\right) d t+d \mathbf{B}_{t}, \text { with } \mathbf{X}_{0} \sim \mathcal{U}(A) .
$$

The drift depends on a function $f: \mathbb{R} \rightarrow \mathbb{R}$, assumed to be continuous and bounded. The term $\mathbf{B}_{t}$ denotes a standard Wiener process. $\mathcal{U}(A)$ is the uniform distribution, over some compact set $A \subset \mathbb{R}$.

The SDE in (9) is discretized in time according to a first-order Euler-Maruyama scheme, with discretization step $\Delta>0$, which yields the following, for any $n \geq 0$ : $\mathbf{X}_{(n+1) \Delta} \sim \mathcal{N}\left(\mathbf{X}_{n \Delta}+\Delta f\left(\mathbf{X}_{n \Delta}\right), \Delta\right)$, where $\mathcal{N}\left(m, \sigma^{2}\right)$ is a normal random variable with mean $m$ and variance $\sigma^{2}$.

For computational necessity, we shall introduce some approximation outside the compact interval $\mathcal{K}=[-K, K]$. Let us partition this interval $\mathcal{K}$ into $2 l$ sections of length $2 \delta$, where $\delta=K / l$, and centered at the representative points $\bar{x}_{k}=-K+$ $(2 k-1) \delta, k=1,2, \ldots, l$. Call these partitions $\mathcal{D}_{k}=[-K+2(k-1) \delta,-K+2 k \delta]$. Additionally, consider two regions for the open intervals $\mathcal{D}_{l b}=(-\infty,-K], \mathcal{D}_{u b}=$ $[K,+\infty)$, "centered" at the points $\bar{x}_{l b, u b}=\{ \pm(K+\delta)\}$. Consider for convenience the extended index set $\mathcal{Q}=\{1,2, \ldots, l, l b, u b\}$. Conditional on $\mathbf{X}_{n \Delta}=\bar{x}_{k}$, for

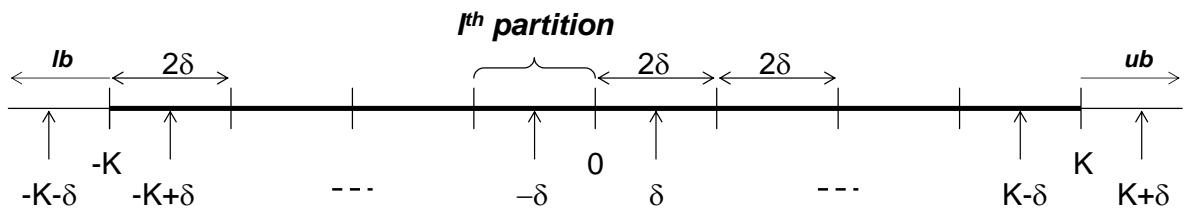

Fig. 1. Abstraction procedure for the one-dimensional system in (9).

any $k \in \mathcal{Q}$, the process at time $(n+1) \Delta$ is distributed according to $T\left(\cdot \mid \bar{x}_{k}\right) \triangleq$ $\mathcal{N}\left(\cdot ; m_{k}, \Delta\right)$, where $m_{k}=\bar{x}_{k}+\Delta f\left(\bar{x}_{k}\right)$.

This discretization procedure induces a dt-SHS, where the $l+2$ domains make up the state space as $\mathcal{S}=\bigcup_{k \in \mathcal{Q}}\{k\} \times \mathcal{D}_{k}$. Let us compute the approximate transition probabilities between the different modes of the introduced dt-SHS based on the representative points, and associate bounds on the errors.

In the following, we implement some computations for the very special lineardrift case, i.e. where $f(x)=-\mu x, \mu>0$. The knowledge of a closed form distribution for this process [11] enables a comparison of it with the outcome of the simulations. We have chosen the following parameters: $K=15, \Delta=1, m=0, \sigma=1$. Choosing a $\mu=0.5$, the solution process of (9) is trivially distributed as $\mathcal{N}(0,1)$. We have implemented our abstraction procedure and the MSC basic algorithms on Matlab. Figure 2 illustrates that, to obtain a precision (say $\varepsilon=0.05$ ) in the steady state computation, we need a MSC abstraction with 50 states. The 
table below Figure 2 shows that by augmenting the state space of the MSC abstraction, the error bounds for the steady state converge to zero.

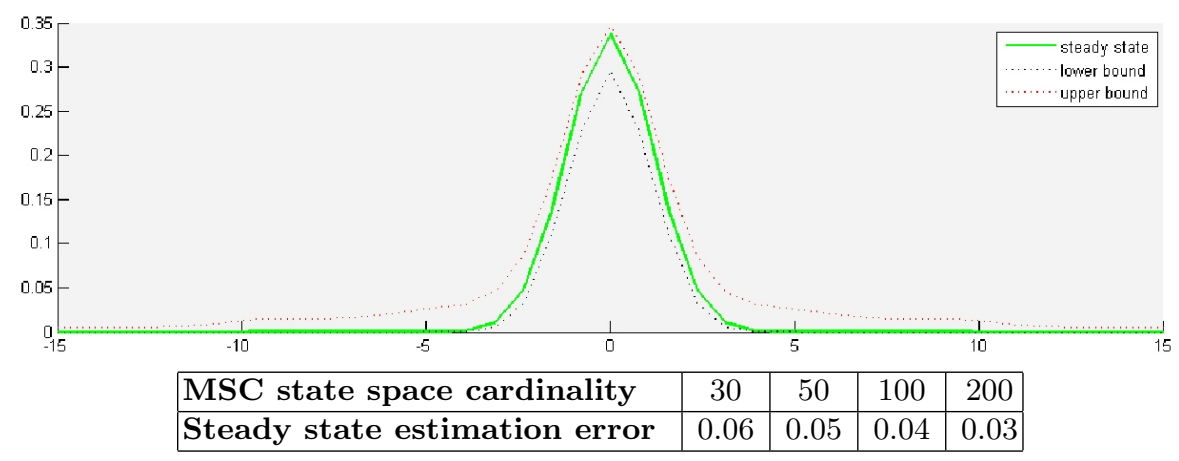

Fig. 2. Simulation outputs.

\section{References}

1. A. Abate, M. Prandini, J. Lygeros, and S. Sastry. Probabilistic reachability and safety for controlled discrete time stochastic hybrid systems. Automatica, 2007. Accepted for publication.

2. R. Alur, T. Henzinger, G. Lafferriere, and G. Pappas. Discrete abstractions of hybrid systems. Proceedings of the IEEE, 88(2):971-984, July 2000.

3. A. Aziz, K. Sanwal, V. Singhal, and R. Brayton. Model-checking continuous time markov chains. ACM Trans. on Comp. Logic, 1(1):162-170, 2000.

4. E.M. Clarke, O. Grumberg, and D.A. Peled. Model Checking. The MIT Press, Cambridge, Massachusetts, 2002.

5. M. H. A. Davis. Markov Models and Optimization. Chapman \& Hall/CRC Press, London, 1993.

6. A. D'Innocenzo, A.A. Julius, M.D. Di Benedetto, and G.J. Pappas. Approximate timed abstractions of hybrid automata. In Proceedings of the $46^{\text {th }}$ IEEE Conference on Decision and Control. New Orleans, Louisiana, USA., 12-14 December 2007.

7. Antoine Girard and George J. Pappas. Approximation metrics for discrete and continuous systems. IEEE Transactions on Automatic Control, 52(5):782-798, 2007.

8. Harald J. Hartfiel. Markov Set-Chains, volume 1695 of Lecture Notes in Mathematics. Springer-Verlag Berlin Heidelberg, 1998.

9. A.A. Julius and G.J. Pappas. Approximate abstraction of stochastic hybrid systems. IEEE Trans. Automatic Control, provisionally accepted.

10. H. J. Kushner. Approximation and Weak Convergence Methods for Random Processes with Applications to Stochastic Systems Theory. MIT Press, Cambridge, Massachussets, 1984.

11. B. Øksendal. Stochastic Differential Equations: An Introduction with Applications. Springer-Verlag, 6th edition, 2003. 